\title{
Constraints on the Intrinsic Charm Content of the Proton from Recent ATLAS Data
}

\section{G. I. Lykasov*}

Joint Institute for Nuclear Research, st. Joliot Curie 6, Dubna, Moscow region, 141980, Russia E-mail: gennady. lykasovecern.ch

\section{S. J. Brodsky}

SLAC National Accelerator Laboratory, Stanford University, Stanford, CA 94025, United States E-mail: sjbtheslac.stanford.edu

\section{A. Bednyakov}

Joint Institute for Nuclear Research, st. Joliot Curie 6, Dubna, Moscow region, 141980, Russia E-mail: vadim.bednyakov@cern.ch

\section{A. V. Lipatov}

Skobeltsyn Institute of Nuclear Physics, Moscow State University 119991 Moscow, Russia

E-mail:artem. lipatov@mail.ru

\section{J. Smiesko}

Comenius University in Bratislava, Faculty of Mathematics, Physics and Informatics Mlynska Dolina, 84248 Bratislava, Slovakia

E-mail:juraj.smiesko@cern.ch

\section{S. Tokar}

Comenius University in Bratislava, Faculty of Mathematics, Physics and Informatics Mlynska Dolina, 84248 Bratislava, Slovakia

E-mail:stanislav.tokarecern.ch

\begin{abstract}
We investigate the possibility to constrain the intrinsic charm probability in proton $w_{\mathrm{c}}$ using first ATLAS data on the associated production of prompt photons and charm-quark jets in $p p$ collisions at $\sqrt{s}=8 \mathrm{TeV}$. The upper limit on the intrinsic charm probability in proton $w_{u l}<1.93 \%$ is obtained at the $68 \%$ confidence level. This constraint is primarily determined by the theoretical scale and systematical experimental uncertainties.
\end{abstract}

Light Cone 2019 - QCD on the light cone: from hadrons to heavy ions September 16-20 2019

Ecole Polytechnique, Palaiseau, France

\footnotetext{
* Speaker.
} 
One of the fundamental predictions of quantum chromodynamics is the existence of Fock states containing heavy quarks at large light-front (LF) momentum fraction $x$ in the LF wavefunctions of hadrons [1,2]. A key example is the $|u u d c \bar{c}\rangle$ intrinsic charm (IC) Fock state of the proton's QCD eigenstate generated by $c \bar{c}$-pairs which are multiply connected to the valence quarks.

The first indication for IC at a high energy collider was observed in the $p \bar{p} \rightarrow \gamma X$ reaction at the Tevatron [3]. Based on our previous results (see [4,5] and references therein), in the present paper we investigate the possibility to constrain the IC probability $w_{\mathrm{c}}$ using first ATLAS data [6] on measurements of differential cross sections of isolated prompt photons produced in association with a $c$-jet in $p p$ collision at $\sqrt{s}=8 \mathrm{TeV}$. The analysis of this effect in detail is presented in our paper [4].

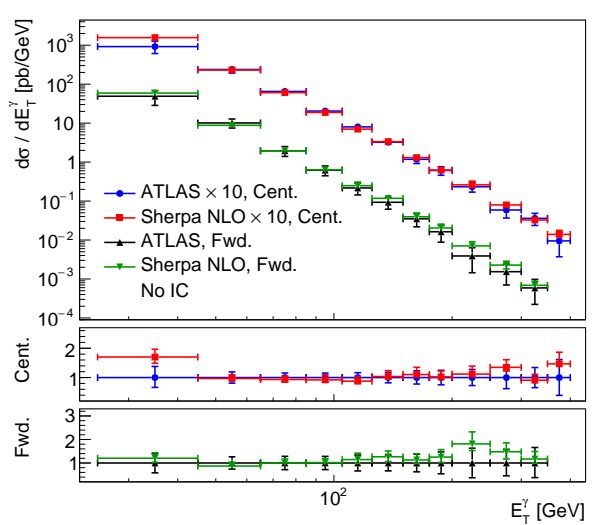

(a) $w_{\mathrm{c}}=0 \%$ (no IC)

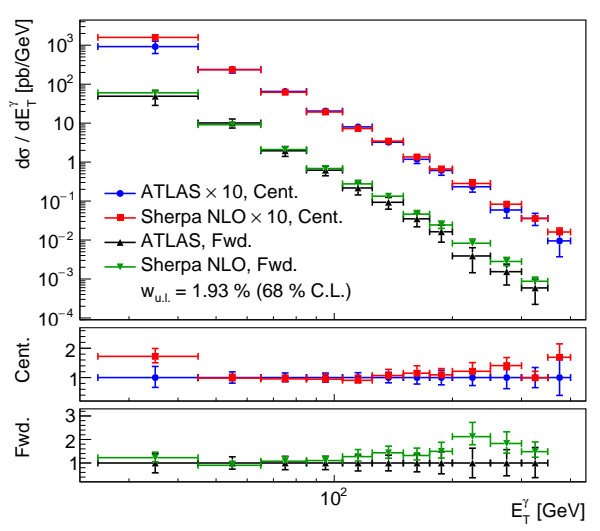

(b) $w_{\mathrm{c}}=w_{\mathrm{u} .1 .}=1.93 \%$

Figure 1: The $E_{\mathrm{T}}^{\gamma}$ spectrum of the $\gamma+c$-jet production calculated with MC generator SHERPA at NLO compared with the ATLAS data [6].

(a) top: the spectrum in the central $\left|\eta^{\gamma}\right|<1.37$ and forward $1.56 \leq\left|\eta^{\gamma}\right|<2.37$ rapidity region without the IC contribution;

(a) middle: the ratio of the $\mathrm{MC}$ calculation to the data in the central rapidity region $\left(w_{\mathrm{c}}=0 \%\right)$;

(a) bottom: the ratio of the $\mathrm{MC}$ calculation to the data in the forward rapidity region $\left(w_{\mathrm{c}}=0 \%\right)$.

(b): the same spectra, as in (a), but with the IC contribution equal to the obtained upper limit $w_{\mathrm{c}}=w_{\mathrm{u} .1 .}=1.93 \%$.

The results on $E_{\mathrm{T}}^{\gamma}$ spectra of the $\gamma+c$-jet production in central and forward regions using the CT14nnloIC [7] parton distribution functions (PDF) without the IC contribution are presented in Fig. 1 (left). One can see from Fig. 1 (left) that the difference between the experimental data and the Monte Carlo (MC) calculation using the generator SHERPA [8] (version 2.2.4) with next-toleading order (NLO) matrix elements are within the total experimental uncertainties. Therefore, we are unable to determine a precise value of the IC probability from recent ATLAS data. However, one can extract an upper limit on the IC contribution to the data. To obtain this limit we employed simple method of fitting MC containing IC to ATLAS data. The resulting upper limit of the IC contribution for SHERPA NLO calculation $w_{\text {u.l. }}=1.93 \%$ at $68 \%$ confidence level (C.L.) is presented in Fig. 1 (right). The details of this calculation are presented in [4].

The $w_{\mathrm{c}}$ extraction method was also repeated with the combined QCD approach instead of 


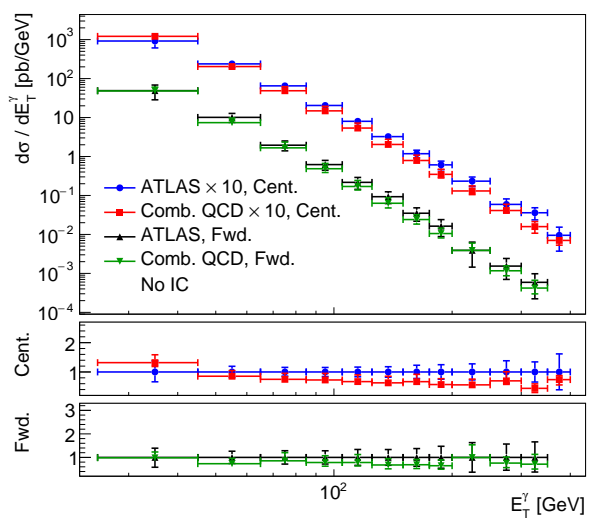

(a) $w_{\mathrm{c}}=0 \%$ (no IC)

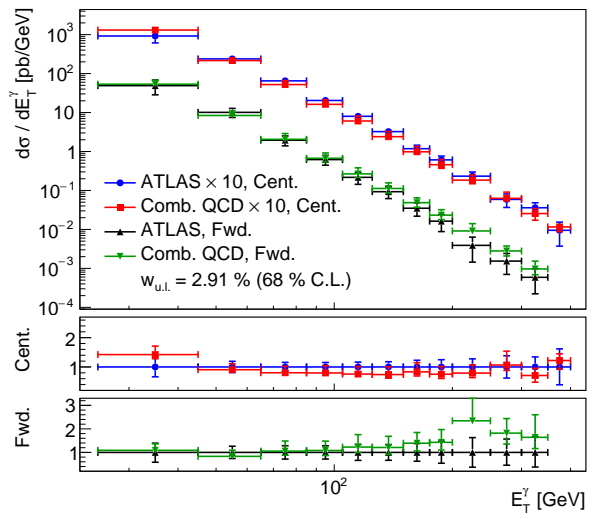

(b) $w_{\mathrm{c}}=w_{\mathrm{u} .1 .}=2.91 \%$

Figure 2: The spectrum of prompt photons associated with $c$-jet as a function of its transverse energy $E_{\mathrm{T}}^{\gamma}$ calculated using the combined QCD approach [4], compared with ATLAS data [6].

(a) top: the spectrum in the central $\left|\eta^{\gamma}\right|<1.37$ and forward $1.56 \leq\left|\eta^{\gamma}\right|<2.37$ rapidity region without the IC contribution;

(a) middle: the ratio of the $\mathrm{MC}$ calculation to the data in the central rapidity region $\left(w_{\mathrm{c}}=0 \%\right)$;

(a) bottom: the ratio of the MC calculation to the data in the forward rapidity region $\left(w_{\mathrm{c}}=0 \%\right)$.

(b): the same spectra, as in (a), but with the IC contribution equal to the obtained upper limit $w_{\mathrm{c}}=w_{\text {u. } 1 .}=2.91 \%$.

SHERPA NLO calculation and the results can be seen in Fig. 2. The combined QCD is based on the $k_{T}$-factorization formalism $[9,10]$ in the small- $x$ domain and the assumption of conventional (collinear) QCD factorization at large $x$. Within this approach, we have employed the $k_{T^{-}}$ factorization approach to calculate the leading contributions from the $\mathscr{O}\left(\alpha \alpha_{s}^{2}\right)$ off-shell gluongluon fusion $g^{*} g^{*} \rightarrow \gamma c \bar{c}$. $\gamma c$ production. In addition to that, we take into account several standard pQCD subprocesses involving quarks in the initial state using the conventional DGLAP-based (collinear) QCD factorization. These are the flavor excitation, quark-antiquark annihilation and quark-gluon scattering subprocesses, which become important at large transverse momenta (or, respectively, at large parton longitudinal momentum fraction $x$. Thus, we apply a combination of two techniques (referred as a "combined QCD approach") employing each of them in the kinematic regime where it is most suitable. More details can be found in [4]) (see also references therein). The upper limit of the IC contribution obtained within the combined QCD approach is $w_{\mathrm{u} .1 .}=2.91 \%$. Note that the combined QCD does not include parton showers and hadronization, a contribution which is sizable at $E_{\mathrm{T}}^{\gamma}>100 \mathrm{GeV}$, where the IC signal could be visible. Therefore, the results obtained from SHERPA NLO calculation, which include these effects, are more realistic.

The precision of our analysis is limited by the experimental systematic uncertainties - mainly, by the $c$-tagging uncertainty which is predominantly connected with the light jet scaling factors [6]. It is also limited by theoretical QCD scale uncertainties. The PDF uncertainties are included in the predictions using the SHERPA NLO. In contrast to these uncertainties, the statistical uncertainty does not play a large role. In Fig. 3 we have shown the fraction of the uncertainty of the IC upper limit $w_{\text {u.l. }}$ at $68 \%$ C.L. introduced from the uncertainty of each contributing component. The 
allowed upper limit is presented four times, every time the component of uncertainty in question is reduced from its actual value (100\%). This assumes that the central values of the experiment does not change.

A first estimate of the intrinsic charm probability in the proton has been carried out utilizing recent ATLAS data on the prompt photon production accompanied by the $c$-jet at $\sqrt{s}=8 \mathrm{TeV}$ [6]. We estimate the upper limit of the IC probability in the proton at $1.93 \%$ with $68 \%$ C.L. In order to obtain more precise results on the intrinsic charm contribution in $\gamma+c$-jet channel one needs additional data at larger $E_{\mathrm{T}}^{\gamma}$ and at the same time reduced systematic uncertainties which come primarily from $c$-jet tagging. Data at different energies at the LHC which check scaling predictions and future improvements in the accuracy of flavor tagging will be important.

The SLAC Pub number is SLAC - PUB - 17505 with title: Constraints on the Intrinsic Charm Content of the Proton from Recent ATLAS Data.

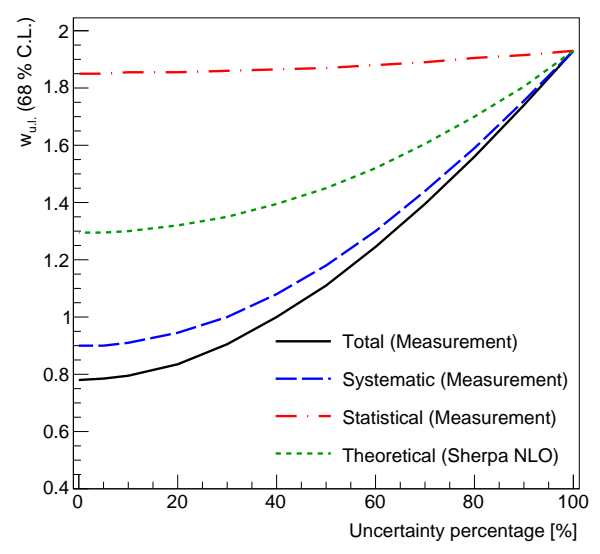

Figure 3: The dependence of the IC probability upper limit $w_{\mathrm{u} .1}$ at $68 \%$ C.L. on the reduction of the particular uncertainty component.

\section{References}

[1] S.J. Brodsky,P. Hoyer, C. Peterson, N. Sakai, Phys.Lett. B 93 (1980) 451.

[2] S.J. Brodsky, C. Peterson, N. Sakai, Phys.Rev. D 23 (1981) 2745.

[3] V.M. Abazov, et al. (D0), Phys.Rev.Lett. 102 (2009) 192002.

[4] V.A. Bednyakov, et al. Eur.Phys.J.C 79 (2019) 92.

[5] S.J. Brodsky, et al. Prog. Part. Nucl. Phys. 93 (2017) 108.

[6] M. Aaboud, et al. (ATLAS), Phys. Lett. B 776 (2018) 295.

[7] T.-J. Hou, et al., JHEP 02 (2018) 059.

[8] T. Gleisberg, et al., JHEP 02 (2009) 007.

[9] S. Catani, M. Ciafaloni, F. Hautmann, Nucl.Phys., B366 (1991) 135.

[10] B.Andersson, et al. (Small x) Eur.Phys.J C 25, 2002) 77.

[11] P.M. Nadolsky, et al., Phys. Rev. D 78 (2008) 013004. 